\title{
Verträge auf elektronischem Weg
}

\author{
Ursula Sury ${ }^{1}$ \\ Online publiziert: 21. Januar 2022 \\ (c) The Author(s), under exclusive licence to Springer-Verlag GmbH Deutschland, ein Teil von Springer Nature 2022
}

\section{Die Ausgangslage}

Durch die anhaltende Covid-19-Pandemie weicht der traditionelle, zentrale Arbeitsplatz zunehmend Home-OfficeLösungen. Der moderne Arbeitsprozess verlagert sich in die digitale Welt und der klassische Briefverkehr weicht der elektronischen Mail. Der virtuelle Austausch von bedeutenden Dokumenten rückt mehr denn je in den Vordergrund.

Dass E-Mails mittlerweile die meistbenutzte Form der Onlinekommunikation sind, überrascht nicht. So drängt sich die Frage auf, ob und wie Verträge auf elektronischem Weg gültig zustande kommen.

\section{Können Verträge „online“ zustande kommen?}

Ein Vertrag kommt grundsätzlich durch übereinstimmende Willensäusserung zustande. Eine Unterschrift auf Papier ist bis auf gewisse gesetzliche Ausnahmen nicht vorausgesetzt.

So werden im Alltag eine Vielzahl von Geschäften mündlich oder sogar stillschweigend abgewickelt. Zum Beispiel kauft jemand am Kiosk eine Tageszeitung und legt wortlos das Geld auf den Tresen. Ähnliches ereignet sich in der digitalen Welt. So klickt eine Nutzerin eine Warenbeschreibung an, überträgt ein Icon in den virtuellen Warenkorb und lässt sich den Gegenstand per Post zustellen. Die übereinstimmende Willenserklärung (die Offerte und deren Annahme) lässt sich oft aus den Umständen ableiten und erstreckt sich dabei auf alle objektiv wesentlichen Vertragsbestandteile, z.B. den Preis der Zeitung am Kiosk oder die Art und Beschreibung des Gegenstands gemäß Online-Shop.

So kann grundsätzlich per E-Mail, Telefon, WhatsApp oder per Mausklick (beispielsweise auf einem Webshop)

\footnotetext{
Ursula Sury

ursula.sury@dieadvokatur.ch

1 Luzern, Schweiz
}

ein Geschäft abgeschlossen werden. Das Geschäft gilt dabei als unter Abwesenden als Vertrag geschlossen.

\section{Wann reicht eine einfache E-Mail nicht aus?}

In der Regel geht es bei auseinanderfallenden Meinungen darum, dass eine Partei beweisen muss, dass ein Geschäft bzw. ein Vertrag überhaupt zustande gekommen ist. Der Austausch von E-Mail oder von Textnachrichten genügt in der Regel als Beweis für einen Vertragsabschluss.

Eine einfache E-Mail ist u. U. unzureichend, wenn an den Vertrag vom Gesetz bestimmte Anforderungen an die Form gestellt werden oder wenn die beteiligten Vertragsparteien eine bestimmte Form vorbehalten.

Oft werden heute Verträge als Kopie mit E-Mail zugestellt, dann ausgedruckt, visiert, gescannt und wieder per E-Mail als Kopie zugestellt. Oft denken dann die Involvierten, dass ein Vertrag formgültig zustande gekommen ist. Viele Behörden akzeptieren gerade solche Übermittlungen nicht, weil Fälschungen oder Fehler in der Übermittlung befürchtet werden.

Daher reicht eine visierte Kopie per E-Mail dann nicht aus, wenn an den Beweis einer Urkunde (in diesem Fall der Vertrag) eine höhere Anforderung gestellt wird oder befürchtet werden muss, dass das Dokument elektronisch verändert werden könnte - z.B. könnte die Identität des Absenders oder der Inhalt manipuliert werden.

\section{Die elektronische Signatur als Lösung?}

Im heutigen Arbeitsleben ist es jedoch unvorstellbar geworden, jeden einzelnen Vertrag physisch zur Unterzeichnung zu versenden. Eine Lösung, welche eine Alternative zur eigenhändigen Unterschrift bietet, stellt die elektronische Signatur dar, welche das Potenzial hat, die Unterschrift auf Papier abzulösen.

Eine elektronische Signatur ist unter gewissen Voraussetzungen der eigenhändigen Unterschrift gleichgestellt. 
Durch ein technisches Verfahren soll die Herkunft eines elektronischen Dokuments (Authentizität) bestimmt werden können und gleichzeitig überprüft werden, ob das Dokument unverändert geblieben ist (Integrität). Der Nachweis der Authentizität und Integrität eines Dokuments werden durch ein Zertifikat einer entsprechenden Anbieterin gesichert.

Es gibt verschiedene Arten von elektronischen Signaturen. Der eigenhändigen Unterschrift ist hingegen nur die sogenannte qualifizierte elektronische Signatur gleichgestellt.

In der EU gilt seit 2016 die eIDAS - electronic IDentification, Authentication and trust Services (Verordnung Nr. 910/2014), die sogenannte Signaturrichtlinie. Die Verordnung regelt die rechtlichen Rahmenbedingungen für eine elektronische Signatur im EU-Raum und sorgt für einheitliche Standards. An diese Verordnung angelehnt ist in der Schweiz im Jahre 2017 das ZertES (Bundesgesetz über die elektronische Signatur) und dessen Verordnung in Kraft getreten.

Ein wesentlicher Unterschied der Schweizer Regelung in Vergleich mit den EU-Signaturrichtlinien liegt darin, dass die jeweiligen Zertifizierungsdienste durch die Schweizerische Akkreditierungsstelle anerkennt werden müssen. Somit ist in der Schweiz nicht nur eine gesetzeskonforme elektronische Signatur notwendig, sondern sie muss auch durch einen anerkannten Zertifizierungsdienst ausgestellt worden sein. Eine Akkreditierung eines Zertifizierungsdienstes bleibt in der EU dagegen freiwillig.

\section{Welche Arten der elektronischen Signatur gibt es?}

Grundsätzlich werden sowohl in der Schweiz als auch der EU drei verschiedene Sicherheitsstufen von Signaturen unterschieden.

Bei der einfachen elektronischen Signatur (ES) handelt es sich um einen Sammelbegriff für alle Signaturen, welche kein fortgeschrittenes oder qualifiziertes Niveau haben. Die eIDAS definiert eine einfache Signatur als „Daten in elektronischer Form, die anderen elektronischen Daten beigefügt oder logisch mit ihnen verbunden werden und die der Unterzeichner zum Unterzeichnen verwendet". Somit ist das digitale „Schreiben“ des Namens, ob in einer E-Mail oder einem Dokument, bereits eine einfache Signatur oder der Mausklick auf einen „Ich stimme zu“-Button eines Online-Formulars.

Im Gegensatz zu einer einfachen elektronischen Signatur muss eine fortgeschrittene elektronische Signatur (AES) vier Anforderungen erfüllen. Sie muss eindeutig dem Unterzeichner/Unterzeichnendem zugeordnet werden können und dessen Identifizierung ermöglichen. Zudem muss die
Signatur unter Verwendung elektronischer Signaturerstellungsdaten erstellt werden und den Integritätsschutz gewährleisten.

Bei der qualifizierten elektronischen Signatur (QES) handelt es sich wiederum um eine fortgeschrittene Signatur, welche auf einem qualifizierten Zertifikat für elektronische Signaturen beruht.

\section{Wann kommt welche elektronische Signatur zum Einsatz?}

Bei der Entscheidung, welche Signatur zur Anwendung gelangen soll, muss zu Beginn sichergestellt werden, ob eine gesetzliche Schriftformerfordernis vorliegt. Falls ein Vertrag an diese Form gebunden ist, muss geprüft werden, ob die gesetzlichen Bestimmungen die Nutzung einer QES erlauben oder, wie bei einer öffentlichen Beurkundung, keine Art von elektronischer Signatur möglich ist.

Die höchste und sicherste Form der elektronischen Unterschrift, die QES, wird aufgrund ihrer hohen Anforderungen nur in ganz bestimmten Fällen eingesetzt, wie beispielsweise im Behördenverkehr.

Sollte keine gesetzliche Formvorschrift vorliegen, ist eine ES nicht immer die richtige Wahl. Es sollten Risiken im Detail analysiert und ein allfälliger Zusatznutzen durch die Verwendung einer elektronischen Signatur abgeklärt werden.

Solange Unterlagen mit einem begrenzten rechtlichen oder finanziellen Risiko verbunden sind, reicht eine ES. Sollte es sich aber um bedeutende Dokumente handeln, wie beispielsweise Gesellschaftsverträge oder Sozialversicherungsdokumente, bringt eine AES ein höheres Sicherheitsniveau als eine ES und garantiert eine verstärkte Beweiskraft, welche bei wichtigen Rechtsgeschäften ausdrücklich zu empfehlen ist. Ein weiterer Vorteil der elektronischen Signatur ist die Zeitersparnis, indem Prozesse deutlich beschleunigt werden können und zahlreiche Zwischenschritte entfallen (Ausdrucken, Unterschreiben, Kopieren, Scannen).

In der Privatwirtschaft kommt vor allem die AES zur Anwendung, solange kein gesetzliches Schriftformerfordernis vorliegt, da die Benutzung vergleichsweise unkompliziert ist.

\section{Fazit}

Der Vertragsschluss auf elektronischem Weg wird täglich praktiziert. Im Einzelfall kann es zu Beweisschwierigkeiten kommen und allenfalls ist der Vertrag ungültig, weil die vom Gesetz oder durch die Parteien vereinbarte Form 
nicht vorliegt. Die elektronische Signatur bietet eine Lösung, die in technischer Hinsicht mehr Sicherheit bieten kann als das typische auf Papier signierte Dokument. Ob es dabei der qualifizierten elektronischen Signatur bedarf, muss im Einzelfall abgeklärt werden. Doch mit der entsprechenden elektronischen Signatur ist für alle Beteiligten nachvollziehbar von wem und wann ein bestimmtes Do- kument mit einem festgeschriebenen und unbestreitbaren Inhalt unterzeichnet wurde.

Hinweis des Verlags Der Verlag bleibt in Hinblick auf geografische Zuordnungen und Gebietsbezeichnungen in veröffentlichten Karten und Institutsadressen neutral. 